\title{
Effects of Ce on Extrusion Alloy Phase and Mechanical Properties of ZM21 Magnesium Alloy
}

\author{
Quan $\mathrm{Li}^{1, \mathrm{a} *}$, Weibo Zhu ${ }^{1}$, Aimin Jiang $^{1}$, Bin Zeng ${ }^{1,2}$ \\ ${ }^{1}$ Chongqing Academy of Science and Technology, Chongqing 401123, China \\ ${ }^{2}$ College of Materials Science \& Engineering, Chongqing University, Chongqing 400030, China \\ a156723583@qq.com \\ Corresponding Author: Quan Li
}

Keywords: Magnesium alloy; Homogenization; Microstructure; Extrusion

\begin{abstract}
In this paper, the effects of Ce addition on extrusion alloy phase and mechanical properties of extruded ZM21 magnesium alloy were investigated by the use of XRD, SEM and tensile test at room temperature et al. Analysis of the cerium magnesium alloy extrusion of ZM21 influence of alloy phase and mechanical properties and mechanism, The results show that of Ce can improves the mechanical properties such as ductility and strength, mainly due to deformation of the second phase in the alloy of Ce element formed by the fine grain structure and stability grain boundary sliding exert influence on the mechanical properties of the alloy when hinder plastic deformation.
\end{abstract}

\section{Introduction}

ZM21 alloy is a kind of Mg-Zn alloy with low levels of alloy, the hot extrusion performance is better than that of AZ31 magnesium alloy, suitable for rapid extrusion to reduce products cost ${ }^{[1-2]}$. German GKSS research center for magnesium alloy ZM21 was realized using the hydrostatic extrusion high-speed extrusion of more than $120 \mathrm{~m}$ per minute ${ }^{[3-5]}$, CAST research centre in Australia holds ZM21 magnesium alloy hot working performance to the attention ${ }^{[6]}$. The influence of rare earth on magnesium alloy has a very good, not only constitutes the-Re Mg alloy series, but also for Mg-Al, Mg-Zn alloy system has a very significant effect, its main functions are: fine grains, purify melt, improve the alloy at room temperature strength, improve the mechanical properties of the alloy thermal stability, improve the alloy corrosion resistance and so on. By adding different content of cerium ZM21 magnesium alloy extrusion experiments, research of cerium on the alloy phase and mechanical properties of ZM21 magnesium alloy extrusion effect and its mechanism, optimization of rare earth element cerium in ZM21 magnesium alloy addition amount, in order to obtain excellent comprehensive mechanical properties of the alloy, and to provide experimental basis.

\section{Experiments}

Alloy raw materials including $\mathrm{Mg}$ (mass fraction 99.3\%), Zn (mass fraction 99.9\%), 0.39 Mg-Mn intermediate alloy and Mg-20Ce intermediate alloy, with low carbon steel crucible in 60 $\mathrm{kW}$ resistance furnace refining, 5 flux as refining agent and coating agent, by a water-cooled semicontinuous casting system cast into casting of $\Phi 92 \mathrm{~mm}$. Melting and pouring process with $\mathrm{CO}_{2}$ and $\mathrm{SF}_{6}$ gas mixture for their own protection. By $\mathrm{Mg}$ successive increase in 2.0 recent $1.0 \mathrm{Mn}$ alloy Mg-20Ce intermediate alloy, access to Mg-2.0Zn-1.0Mn for the substrate, the laser spectrometer detection component as shown in table 1 . Will cast the sample in the $12 \mathrm{kw}$ box-type resistance furnace of wind circulation homogenization processing, $12 \mathrm{~h}$ under $420^{\circ} \mathrm{C}$ temperature annealing; And then, in 500 tons of extrusion production test horizontal extrusion machine, $400^{\circ} \mathrm{C}$ preheat $2 \mathrm{~h}$,

extrusion temperature is $400^{\circ} \mathrm{C}$, extrusion ratio for 28 , extrusion products $\Phi 16 \mathrm{~mm}$ diameter. Squeezed state sample direct interception on extrusion rods, picric acid solution after corrosion,

Table 1. Chemical component of the alloy ( wt\%) 
the Rigaku D/Max-1200 type X-ray diffractometer (alpha) Cu target, $\mathrm{K}$ phase analysis. Using TESCAN VEGA company II LMU variable vacuum SEM and EDS tissue morphology scan and compound composition analysis. Think twice in the new CMT-5105 electronic universal material testing machine room temperature tensile test, the tensile rate of $4 \mathrm{~mm} / \mathrm{min}$.

\begin{tabular}{ccccc}
\hline Alloy & \multicolumn{4}{c}{ Alloy chemical composition } \\
Number & Zn & Mn & Ce & Mg \\
\hline $1 \#$ & 1.80 & 0.95 & 0 & other \\
2\# & 1.92 & 0.91 & 0.19 & other \\
3\# & 2.12 & 0.93 & 0.28 & other \\
4\# & 2.06 & 0.92 & 0.39 & other \\
5\# & 2.14 & 0.91 & 0.57 & other \\
\hline
\end{tabular}

Test results and analysis
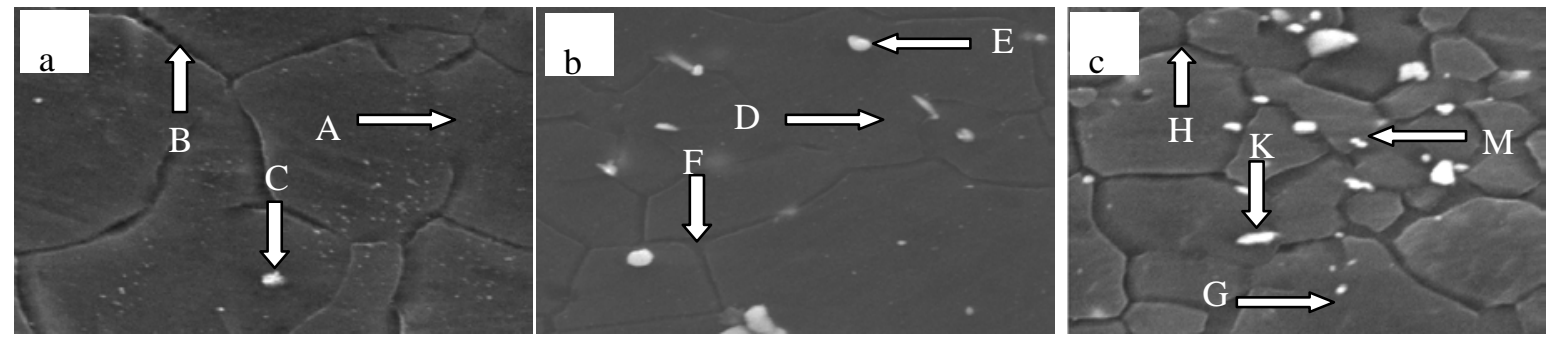

Fig.1 EDX results of extruded alloy (a) 1\#; (b) 3\#; (c) 5\#;

Choose 1\#, 3\#, 5\# alloy energy spectrum analysis, the energy spectrum analysis is shown in figure 1, each testing point elements atomic scores are listed in table 2, all sorts of figure 2 for adding rare earth Ce ZM21 magnesium alloy extrusion state X-ray diffraction phase analysis. 1\# alloy angular second phase Mn matrix and near grain boundary is the atomic content of Mn content of 3-4 times, show that the phase of rich manganese phase, combined with XRD analysis for Mn; And near grain boundary in the matrix containing zinc and Mn element, and their levels are basically the same in different position, the second phase should be no solid soluble in matrix form. $3 \#$ alloy matrix, near grain boundary element and the second phase and Ce, and near grain boundary Ce content is Ce in the matrix content of 2 times, and the second phase of Ce content of 4 times.

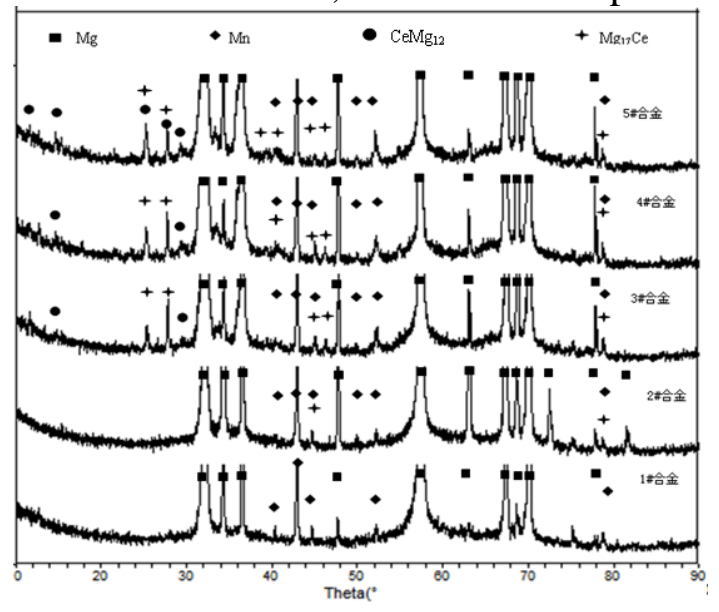

Fig2 XRD of extruded alloys 
Tab2 EDX results of extruded alloy（at, \%)

\begin{tabular}{ccccc}
\hline location & $\mathrm{Mg}$ & $\mathrm{Mn}$ & $\mathrm{Zn}$ & $\mathrm{Ce}$ \\
\hline $\mathrm{A}$ & 98.86 & 0.24 & 0.91 & \\
B & 98.81 & 0.33 & 0.86 & \\
$\mathrm{C}$ & 96.57 & 0.92 & 0.53 & \\
$\mathrm{D}$ & 98.67 & 0.49 & 0.83 & 0.01 \\
E & 98.78 & 0.37 & 0.80 & 0.04 \\
F & 98.37 & 0.56 & 1.05 & 0.02 \\
G & 98.71 & 0.47 & 0.82 & \\
H & 94.69 & 0.93 & 3.43 & 0.96 \\
K & 98.69 & 0.54 & 0.77 & \\
M & 97.53 & 0.39 & 1.78 & 0.3 \\
\hline
\end{tabular}

Near grain boundary Mn content is relatively high, so is zinc. Ce of 5 \# alloy are mainly distributed in the second phase $(\mathrm{M})$ and near grain boundary point $(\mathrm{H})$; In addition to the part of the solution $(\mathrm{G})$, Mn and distribution in the second phase $(\mathrm{K})$ and $(\mathrm{H})$ near grain boundary; Zinc has a similar phenomenon. A small amount of solid soluble in matrix, the rest distributed in the second phase and near grain boundary, and its content is several times that of the matrix.

Squeezed state alloy's mechanical properties are shown in table 3, after extrusion, alloy tensile strength, yield strength were improved significantly, especially the yield strength of as-cast less than 80 mpa to 160 mpa above, 2 \#, 5 \# alloy is to reach more than $180 \mathrm{mpa}$, including 5 \# alloy is close to $200 \mathrm{mpa}$. And as-cast first decreases gradually increase after different, alloy extrusion state with the increase of the content of Ce its strength rising; After adding Ce, plastic alloy are greater than 1 \# alloy without adding Ce. 4 \# and 5 \# alloy plasticity and strength of the highest, respectively. After homogenizing annealing, from Mn precipitation phase in the alloy, the precipitated phase within the grain boundary and grain distribution in solid solution, but due to the precipitation of the second phase is pure Mn, strengthen the effect of alloy. Besides $\mathrm{Mg}$ - Ce and Ce - zinc from the alloy phase partly dissolved, the remnants of $\mathrm{Mg}$ - Ce and $\mathrm{Ce}$ - zinc is basic same, cerium and magnesium, to form the intermetallic compound $\mathrm{Mg}$ - Ce, distributed in the intracrystalline and grain boundary, strengthened the grain boundary, later in the plastic deformation process, the broken are dispersed in matrix of zn-al alloy, is conducive to strengthening alloy. Most ZM21 magnesium alloys of rare earth elements exist in the second phase, the influence of rare earth on the plastic is two-sided, distributed in the second phase of grain boundary have the function of the stability of fine grain structure, good for plastic deformation, at the same time, the rare earth hinder the plastic deformation when the second phase of grain boundary sliding, and generate stress concentration against further plastic deformation. 2 \# alloy with the adding amount of rare earth Ce and its tiny, grain refinement effect is greater than the rare earth in the second phase of the impact of the obstacles to plastic deformation when the effects of the grain boundary sliding, so the plastic to improve; And 3 \# alloy although small grains than 2 \# alloy, but because of the increased concentration of rare earth in the second phase, its brings the function also increase to the detriment of the plastic, plastic unfavorable factors to dominate, so there was no plastic instead of 2 \# alloy; 4 \# alloy rare earth second relative plastic favourable dominant on the one hand, the plastic and to increase; When rare earth content reached 5 \# alloy, rare earth balance two aspects of the influence of the relative plastic, plastic, no longer maintain the level of 4 \# alloy.

Tab.3 Mechanical properties of the alloys

\begin{tabular}{ccccccc}
\hline \multirow{2}{*}{ alloy } & \multicolumn{2}{c}{ UTS/Mpa } & \multicolumn{2}{c}{ YS/Mpa } & \multicolumn{2}{c}{ Elongation/\% } \\
\cline { 2 - 7 } & cast & extruded & cast & extruded & cast & extruded \\
\hline 1\# & 194 & 259 & 60 & 168 & 21.5 & 20.9 \\
$2 \#$ & 187 & 265 & 53 & 186 & 14.7 & 22.9 \\
3\# & 191 & 269 & 61 & 187 & 18.0 & 21.3 \\
$4 \#$ & 198 & 272 & 71 & 195 & 18.0 & 24.1 \\
$5 \#$ & 200 & 276 & 74 & 199 & 20.0 & 24.0 \\
\hline
\end{tabular}




\section{Conclusions}

Add Ce of ZM21 magnesium alloy matrix elements have granular in the second phase, and with the increase of Ce content, gradually increase the number of the second phase, at the same time alloy grain size became smaller, the second phase particle size and the ratio of the alloy grain size increases gradually.

After extrusion, along with the increase of the content of Ce alloy tensile strength, yield strength has been greatly improved, especially the yield strength of as-cast less than 80 mpa to 160 mpa above, plastic alloy were greater than that of without adding Ce ZM21 magnesium alloy.

ZM21 magnesium alloys of rare earth element Ce most exists in the second phase (Mn, MgCe12 and Mg17Ce2), broken and granular MgCe12 Mg17Ce2 phase distribution between the intracrystalline and grain boundary, the deformation of the alloy by stable fine grain and obstacles in the process of plastic deformation when the grain boundary sliding to influence the mechanical properties of the alloy. When Ce content reached $0.39 \%$, the second relatively rare earth plastic influence two aspects reach balance, plastic no longer increase.

\section{Acknowledgements}

This work is supported by the International Science and Technology Cooperation Project (2013DFA71070,2014DFG52810); Chongqing integrated demonstration projects (cstc 2013 jcsf 50003);

\section{References}

[1] Yu Kun, Li Wenxian, Zhang Shijun. Mechanism of Grain Refining by Adding Cerium in Mg and Mg Alloys[J]. Rare Metal Materials and Engineering, 2005, Vol.34(7):1013-1016.

[2] Ravi Kumar N V, Blandin 1 J, Desrayaud C et al. Grain refinement in A791 magnesium alloy during thermomechanical processing(J]. Materials Science and Engineering, 2003, A359: 150-157.

[3] Bussiba A, Ben Artzy A, Shtechman A et al. Grain refinement of AZ31 and ZK60 Mg alloys-towards superplasticity studies[J]. Materials Science and Engineering, 2001, A302: 56-62.

[4] J.S. Zhang, J. Yan, W. Liang, C.X. Xu, C.L. Zhou. Icosahedral quasicrystal phase in Mg-Zn-Nd ternary system [J]. Materials Letters, 62 (2008): 4489-4491.

[5] Li Wenxian. Magnesium and magnesium alloys[M]. Changsha:Central South University Press, 2005.6.

[6] Pan Fusheng, Han Enhou. High-Performance Wrought Magnesium Alloy and Their Processing Technigues[M]. Beijing: Science Press, 2007.18. 